\title{
Correlation of Climate Factors with the COVID-19 Pandemic in USA
}

\author{
Muhammad Mohsinul Hoque*, Umme Saima, Sadia Sultana Shoshi \\ Department of Statistics, Noakhali Science and Technology University, Noakhali, Bangladesh \\ Email address: \\ mmhtc1698@gmail.com (M. M. Hoque), saimaumme@yahoo.com (U. Saima), sadiashoshi0110@gmail.com (S. S. Shoshi) \\ ${ }^{*}$ Corresponding author
}

\section{To cite this article:}

Muhammad Mohsinul Hoque, Umme Saima, Sadia Sultana Shoshi. Correlation of Climate Factors with the COVID-19 Pandemic in USA. Biomedical Statistics and Informatics. Vol. 5, No. 3, 2020, pp. 65-69. doi: 10.11648/j.bsi.20200503.12

Received: July 17, 2020; Accepted: August 17, 2020; Published: September 3, 2020

\begin{abstract}
The coronavirus diseases 2019 (COVID-19) caused by a novel coronavirus which was first identified in Wuhan, a city of Eastern China. What began as an epidemic mostly constrained to China has now become a worldwide pandemic. Previous studies on airborne viruses comparable to coronavirus show that there is a notable relationship between the climate indicators and transmission of viruses. This study was conducted to observe the relationships of climate factors on the number of cases and the number of deaths by Coronavirus Disease -19 (COVID-19) and the spread of it in the USA. The datasets for this research have been collected from the regular updates of The New York Times, weather2visit, Current results (weather and science fact), and Americas Health Rankings from 1 April 2020 to 30 April 2020. Findings of our study observed that the highest number of infected people and death was found in New York in April 2020. Kendall's tau-b and Spearman test of correlation reveals that minimum temperature, average temperature, absolute humidity, and air pollution are significantly correlated with the transmission of this virus and the sum of deaths by COVID-19. The verdict of this article will boost the native and international health organizations to understand the spread of COVID-19 in different environmental situations.
\end{abstract}

Keywords: COVID-19, United States of America (USA), April, Climate

\section{Introduction}

Coronavirus is the name of a worldwide frenzy now. As of May 2, 2020, 3233191 positive cases and 227489 deaths in 215 countries on account of coronavirus (COVID-19), had been accounted for around the world [1]. Coronaviruses (CoVs) are the members of the family Coronaviridae, are encompassed infections by a solitary formula and its size within 26-32 kilobases, that is the biggest acquainted genome because of an RNA infection [2]. In December 2019, patients giving viral pneumonia because of an unidentified microbial operator were accounted for in Wuhan, China. An epic coronavirus was along these lines recognized as the causative pathogen. For finding the origin host of this virus a phylogenetic analysis was driven and it suggests that bats may be the first host of this infection, a creature sold at the seafood market in Wuhan may speak to the middle of the road have encouraged the development of the infection in people [3]. A trademark that makes this virus much increasingly perilous is its transformation. The virus has just changed a few times and no one can guess how frequently it will transform and get more grounded. Researchers have comprehended for quite a long time that environmental change would change the manner in which infections spread. As wind speed, dampness, temperature, humidity are basic in the transmission of irresistible illnesses so atmosphere conditions are named apex indicators of coronavirus sicknesses [4]. Although coronaviruses are thought to be sensitive to heat but low temperature is positively related to the quantity of COVID-19 status at what time the temperature is underneath $3^{\circ} \mathrm{C}$ and in this case, no testimony upholding the tallies of COVID-19 which could decay wherein the climate gets thermal [5]. In that case, at extreme temperatures and extreme relative humidity infection suitability was quickly amiss. The SARS coronavirus is dependable at lower temperatures and lower humidity conditions that may encourage its transmission in the society [6]. Notwithstanding the Infection of coronavirus owing to connect to high mortality and its transmission systems stay deficiently comprehended. As no official medicine is not discovered yet for this virus, so prevention is the best way to reduce the transmission from human to human that includes social distancing, maintaining hygiene, covering mouth and nose 
with a mask, washing hands often, cover coughs and sneezes. In this pandemic The USA has the most elevated demise motion because of the coronavirus disease all over the world.

\section{Research Methodology}

United States of America is a country with 50 states. It has a land area of $9,834,000 \mathrm{~km}^{2}$ with a population of 328.2 million. The GDP of the USA is 20.54 trillion USD [8]. The first confirmed case of coronavirus in the USA was found in Washington state on $21^{\text {st }}$ January, 2020 in a traveler from Wuhan. (Holshue, 2020) The epidemic began in Wuhan, China about a month earlier. At the beginning of the April total number of patients was 213,656 and at the end of the month, the number of total patients was 1062508. Dataset for COVID-19 is taken from a regular updates from The New York Times [9] April 1, 2020 - April 30, 2020. The data of climate indicators include maximum temperature, minimum temperatures, average temperatures, humidity, daily sunshine, precipitation, wind-speed, and Air pollution is taken from weather2visit [10], Current Results (weather and science fact) [11] and Americas Health Rankings [12]. Absolute Humidity $(\mathrm{AH})$ in $\mathrm{g} / \mathrm{m}^{3}$ was calculated using the Clausius Clapeyron equation [13] described as follows.

$$
\mathrm{AH}=\frac{6.112 * e^{\left(\frac{17.67 * T}{T+243.5}\right)} * R H * 2.1674}{273.15+T}
$$

As the information was not normally distributed in this manner Kendall and Spearman rank correlation tests were used to look at the connection between factors. SPSS 26 and Microsoft Excel is used to analyze the data.

\section{Result}

\subsection{The Number of Total New Cases and Deaths in the Month of April}

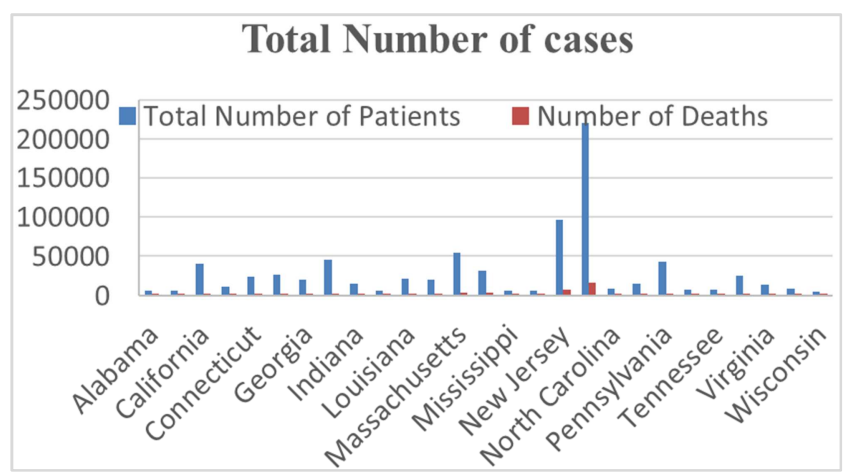

Figure 1. Number of new cases in April.

Figure 1 reports the number of total new patients and the number of total new deaths in different states of the US in April, 20. New York has the highest number of patients and deaths in April 2020. 220, 512 new cases, and 16380 new deaths are recorded in New York in April. New Jersey has the second-highest number of patients (96397) and deaths (6873) in April.

\subsection{Relation Between Meteorological Factor Temperature and Covid-19 Spread and Death}

Table 1 indicates the empirical estimation of Kendall's correlation to test the relationships of meteorological factors with the number of new cases and death by COVID-19. From this table, we identified that minimum and the average temperature is significantly correlated with the number of total new cases and the number of total deaths by COVID-19 in April 2020. The table revealed that minimum temperature (in ${ }^{\circ} \mathrm{C}$ ) had a significant correlation $(0.235, \mathrm{p}=0.020)$ with the occurring of new cases of COVID-19 and also had a significant correlation $(0.234, p=0.021)$ with the deaths of COVID-19. We also found that average temperature (in ${ }^{\circ} \mathrm{C}$ ) had a significant correlation $(0.237, \mathrm{p}=0.015)$ with the occurring of new cases of COVID-19 and also had a significant correlation $(0.207, p=0.034)$ with the deaths of COVID-19.

Table 2 indicates that minimum temperature (in ${ }^{\circ} \mathrm{C}$ ) had a significant correlation $(0.330, \mathrm{p}=0.020)$ with the occurring of new cases of COVID-19 and also had a significant correlation $(0.313, p=0.028)$ with the deaths of COVID-19. We also found that average temperature (in ${ }^{\circ} \mathrm{C}$ ) had a significant correlation (0.329, $\mathrm{p}=0.020)$ with the occurring of new cases of COVID-19 and also had a significant correlation (0.283, $\mathrm{p}=0.046$ ) with the deaths of COVID-19 when we used Spearman's correlation test.

Figure 2 shows the simple bar chart of average temperature by states. Here, from April 1 to April 30, 2020, we have seen that Hawaii, the state of the USA had the maximum average temperature which is $23.11^{\circ} \mathrm{C}$.

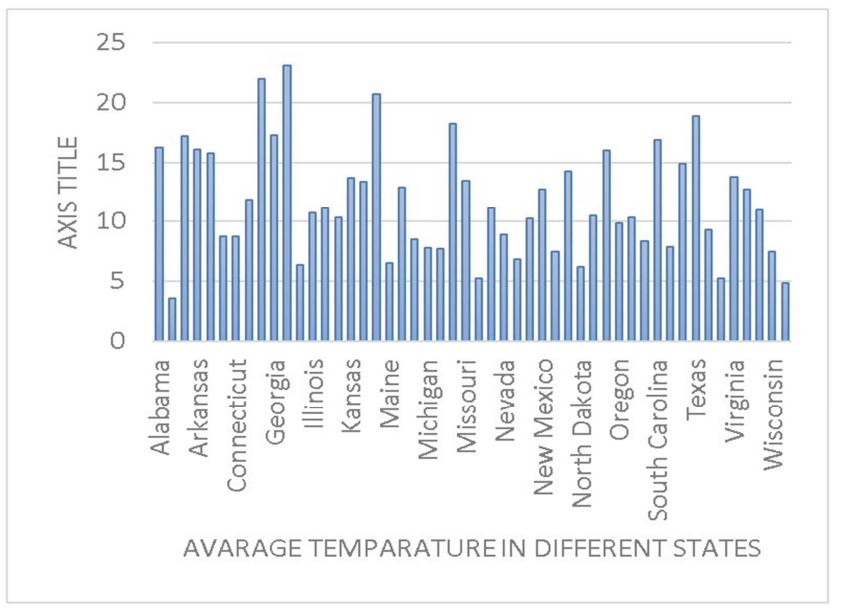

Figure 2. Bar Chart of Average Temperature by states.

\subsection{Relation Between Meteorological Factor Absolute Humidity and Covid-19 Spread and Death}

Table 1 shows that absolute humidity had a significant correlation $(0.211, \mathrm{p}=0.030)$ with the occurring of new cases of COVID-19 and also had a significant correlation (0.181, $\mathrm{p}=0.063$ ) with deaths of COVID-19 when we used Kendall's correlation test. 
Table 2 shows that absolute humidity had a significant correlation $(0.325, \mathrm{p}$ value $=0.021)$ with the occurring of new cases of COVID-19 and also had a significant correlation (0.281, $\mathrm{p}=0.048)$ with deaths of COVID-19 when we used Spearman's correlation test.

\subsection{Relation Between Meteorological Factor Air Pollution and Covid-19 Spread and Death}

Table 1 indicates air pollution had a significant correlation (0.362, $\mathrm{p}=0.000$ ) with the occurring of new cases of COVID-19 and there had no significant correlation between air pollution and death of COVID-19 when we used Kendall's correlation test.

Table 2 indicates that air pollution had a significant correlation $(0.513, \mathrm{p}=0.000)$ with the occurring of new cases of COVID-19 and also had no significant correlation $(0.506$, $\mathrm{p}=0.000$ ) with the deaths of COVID-19 when we used Spearman's correlation test.

Table 1. Kendall's correlation coefficient.

\begin{tabular}{lll}
\hline Climate Variable & Total Cases in April & Total Deaths in April \\
\hline Maximum Temperature & .098 & .008 \\
Minimum Temperature & $.235^{*}$ & $.234^{*}$ \\
Average Temperature & $.237^{*}$ & $.207^{*}$ \\
Daily Sunshine & .019 & .031 \\
Absolute Humidity & $.211^{*}$ & .181 \\
Precipitation & .158 & .138 \\
Wind-speed & .020 & .027 \\
Air Pollution & $.362^{* *}$ & $.353^{* *}$ \\
\hline
\end{tabular}

$* * *, * *, *$ indicates $1 \%, 5 \%$ and $10 \%$ level of significance respectively.

Table 2. Spearman's correlation coefficient.

\begin{tabular}{lll}
\hline Climate Variable & Total Cases in April & Total Deaths in April \\
\hline Maximum Temperature & .152 & .126 \\
Minimum Temperature & $.330^{*}$ & $.313^{*}$ \\
Average Temperature & $.329^{*}$ & $.283^{*}$ \\
Daily Sunshine & .015 & .037 \\
Absolute Humidity & $.325^{*}$ & $.281^{*}$ \\
Precipitation & .228 & .202 \\
Wind-speed & .045 & .054 \\
Air Pollution & $.513 * *$ & $.506^{* *}$ \\
\hline
\end{tabular}

$* * *, * *, *$ indicates $1 \%, 5 \%$ and $10 \%$ level of significance respectively.

\section{Discussion}

From this survey, we found that for the number of total cases in April, minimum temperature and average temperature are significant and for the number of total deaths in April, the average temperature is significant when we use the Kendall correlation test. For Spearman's correlation test, we get the same thing. For the number of total cases in April, minimum temperature and average temperature are significant but for the number of total deaths in April, minimum temperature and average temperature are significant. A previous study which is published in New York City supports our findings. Their findings revealed that for the number of total cases, average temperature and minimum temperature are significant and for the number of total deaths, the average temperature is significant when they used the Kendall correlation test. For the number of total cases and deaths, the average temperature is significant when they used the Spearman rank correlation test [4]. Sonal Gupta, Gourav Singh Raghuwanshi, Arnab Chanda led a review for refining the association between the climate factor temperature and expansion of COVID-19. They observed that in the range of $4^{\circ} \mathrm{C}<\mathrm{T}<11^{\circ} \mathrm{C}$, the expansion of COVID-19 was significantly upheld which is proved that COVID-19 has a relationship with temperature [14]. Another survey was conducted in 2010 about finding the impacts of air temperature on coronavirus endurance on surfaces using two effective representatives which were assessed in that survey utilizing to set up the impacts of air temperature on the continuance of coronavirus on tempered metal. The outcomes of their study show that irresistible virus endured for up 1 to 4 weeks at the temperature of $4^{\circ} \mathrm{C}$. [15]. A research published in Hindawi Publishing Corporation publication where they studied about the durability of the virus at various temperatures on mellifluous surfaces. In their investigation, they have shown that SARS CoV can endure at least fourteen days after absorbing at a temperature found in the air condition environment [6]. Analyzing the impact of temperature within 1 to 2 weeks of the case on COVID-19 spread, a paper was published in Turkey. They revealed that the number of COVID-19 cases is high on a day when the temperature is low on that day [16].

Figure 2 shows that Hawaii which is the state of the USA has the maximum average temperature. As we have seen from our findings that minimum temperature has a significant relationship with COVID-19 spread and all previous results proved that, so the state of Hawaii has the lowest risk of being infected by COVID-19.

From table 1 of this study we also see that when we used Kendall's Correlation test, for the number of total cases in April, absolute humidity is significant and when we used Spearman's correlation test, for the number of total cases and the number of total deaths in April, absolute humidity is significant. Basically, the respiratory disease is raised during rarely cold and low humidity conditions [17]. A survey suggested that absolute humidity is a critical outlines for human the influenza outcomes and lower humidity causes death by affecting influenza virus [18]. Another finding shows that when absolute humidity decreases, then the rate of mortality of COVID-19 increases [19]. Researcher Jeffrey Shaman and Melvin Kohn revealed that the influenza infection endurance rate expanded extraordinarily as per the diminishing of absolute humidity [20]. A study posited that lower humidity was associated with the hazard of primary human cases of MERS-COV. It is also claimed that the humidity is predictable with the association between environment and other respiratory infections [21]. A study claimed that rather than relative humidity, absolute humidity is significantly correlated with influenza epidemic activity [22].

From the outcomes of our survey, we found that air pollution is significant for the total cases and for the total 
deaths in April when we use Kendall's correlation test. For Spearman's correlation test, we found that air pollution is significant for total cases and for total deaths. Statistical analysis on infected individuals on a sample of $\mathrm{N}=50$ Italian province shows that the accelerate of COVID-19 in North Italy has an extraordinary relationship with Air Pollution of urban areas. Which was estimated with days surpassing the cutoff points for PM10 or ozone [23]. In this specific situation, one of the essential things is to explain how the quality of air can impact viral diffusion on the overall levels [24]. In advanced countries, like the US, have more pollution because of temperature inversion. During winters the moistness in all around from a dense haze by mixing with ambient pollution which affects the health of the people [25]. Hot and radiant climate builds temperature and improves air circulation in a condition that can reduce air contamination, and as a result lighten transmission of COVID-19 [26]. Sunlight helps to makes vitamin D which improves the immune system of ours. So in the summer season, we have a greater immune system which helps to adapt Coronaviruses [27].

Therefore, our study demonstrated that minimum temperature, average temperature, absolute humidity and, air pollution are associated with the quantity of all-out new cases and total deaths in 50 states of the US in the month of April. These factors are analytical factors for the COVID-19 transmission. This research on the impact of climate COVID-19 is still particularly bound, so this will add to attempt COVID-19 conditions.

This investigation has a few limitations which ought to be recognized. Though there are countless conceivable meteorological factors experienced for the COVID-19, but just a few factors were considered for this study. Because of the time limitation, we could not take all possible factor which is related to COVID-19. Besides this meteorological factor, there have some other issues which are related to this pandemic spread such as social distancing, government provision, medical facility along with many more things. We hope these questions will be answered in upcoming findings. Furthermore, this outcome may not reflect the real cases of all states. There might have been some error in the data of all states. However, this overview is a preliminary analysis. The fruitful conclusion must have a great period of time and a more exact informational index.

\section{Conclusion}

Climate factors play an important role to combat against the COVID-19 pandemic in the USA. This investigation uncovers that minimum temperature and average temperature have essentially corresponded with the quantity of infected cases and the count of death by COVID-19. The study also reveals that absolute humidity and air pollution are also significantly correlated with the COVID-19 pandemic in the USA for April, 2020. The significance of air pollution indicates that, with the rise in air pollution the COVID-19 pandemic also increases in the USA. The correlation between coronavirus and air pollution means that the crucial part of easing lockdown is to tackle the air pollution. Further research is needed on the spread of COVID-19 considering peoples mobility, lockdown condition, and other climate indicators in the USA.

\section{References}

[1] W. H. Organization, "Coronavirus disease (COVID-19) Pandemic," World Health Organization, 2020. https://www.who.int/emergencies/diseases/novel-coronavirus2019.

[2] S. Su et al., "Epidemiology, Genetic Recombination, and Pathogenesis of Coronaviruses," Trends Microbiol., vol. 24, no. 6, pp. 490-502, 2016, doi: 10.1016/j.tim.2016.03.003.

[3] R. Lu et al., "Genomic characterisation and epidemiology of 2019 novel coronavirus: implications for virus origins and receptor binding," Lancet, vol. 395, no. 10224, pp. 565-574, 2020, doi: 10.1016/S0140-6736(20)30251-8.

[4] M. F. Bashir et al., "Correlation between climate indicators and COVID-19 pandemic in New York, USA," Sci. Total Environ., vol. 728, p. 138835, 2020, doi: 10.1016/j.scitotenv.2020.138835.

[5] J. Xie and Y. Zhu, "Association between ambient temperature and COVID-19 infection in 122 cities from China," Sci. Total Environ., vol. 724, p. 138201, 2020, doi: 10.1016/j.scitotenv.2020.138201.

[6] K. H. Chan, J. S. M. Peiris, S. Y. Lam, L. L. M. Poon, K. Y. Yuen, and W. H. Seto, "The effects of temperature and relative humidity on the viability of the SARS coronavirus," Adv. Virol., vol. 2011, 2011, doi: 10.1155/2011/734690.

[7] I. Business, "Limited coronavirus testing in the US has meant its death rate appears alarmingly high — but it will likely drop," Business Inside, 2020. https://www.businessinsider.com/us-worlds-highest-coronavir us-death-rate-limited-testing-2020-3.

[8] D. Bea, "Gross Domestic Product," BEA Data. https://www.bea.gov/data/gdp.

[9] T. The New York, "Coronavirus in the U. S.: Latest Map and Case Count," The new york times. https://www.nytimes.com/interactive/2020/us/coronavirus-uscases.html?fbclid=IwAR1 wvO0KeOism5VdcNfZmHlw1eDq 2hSv62AbHK_ipfDW8iTIbswfFt65DZ0.

[10] V. Weather2, "United States average temperatures \& weather," Weather2visit, 2020. https://www.weather2visit.com/north-america/united-states.

[11] R. Current, "USA State-Wide Weather Averages," Current Results.

https://www.currentresults.com/Weather/US/average-state-we ather.php?fbclid=IwAR3bmDZLjz2c6z_O-UooM3n8wz7sX0 2ebHVOBo-eWH9PpsyQHMmTolfqvew.

[12] A. H. Rankings, "Explore Air Pollution in California," America's Health Rankings. https://www.americashealthrankings.org/explore/annual/meas ure/air/state/CA/compare/WA?fbclid=IwAR0BxVyKyT8Yz4 WmedvfgU-WSvfXRSu3UslR4vZZYeNxSBEoqB5le3dhYDY. 
[13] Carnotcycle, "How to convert relative humidity to absolute humidity," carnotcycle. https://carnotcycle.wordpress.com/2012/08/04/how-to-convert -relative-humidity-to-absolute-humidity/.

[14] S. Gupta, G. S. Raghuwanshi, and A. Chanda, "Effect of weather on COVID-19 spread in the US: A prediction model for India in 2020," Sci. Total Environ., vol. 728, p. 138860, 2020, doi: 10.1016/j.scitotenv.2020.138860.

[15] L. M. Casanova, S. Jeon, W. A. Rutala, D. J. Weber, and M. D. Sobsey, "Effects of air temperature and relative humidity on coronavirus survival on surfaces," Appl. Environ. Microbiol., vol. 76, no. 9, pp. 2712-2717, 2010, doi: 10.1128/AEM.02291-09.

[16] M. Şahin, "Impact of weather on COVID-19 pandemic in Turkey," Sci. Total Environ., vol. 728, 2020, doi: 10.1016/j.scitotenv.2020.138810.

[17] R. E. Davis, E. Dougherty, C. McArthur, Q. S. Huang, and M. G. Baker, "Cold, dry air is associated with influenza and pneumonia mortality in Auckland, New Zealand," Influenza Other Respi. Viruses, vol. 10, no. 4, pp. 310-313, 2016, doi: 10.1111/irv.12369.

[18] A. I. Barreca and J. P. Shimshack, "Absolute humidity, temperature, and influenza mortality: 30 years of county-level evidence from the united states," Am. J. Epidemiol., vol. 176, no. SUPPL. 7, pp. 114-122, 2012, doi: 10.1093/aje/kws259.

[19] Y. Ma et al., "Effects of temperature variation and humidity on the death of COVID-19 in Wuhan, China," Sci. Total Environ., vol. 724, p. 138226, 2020, doi: 10.1016/j.scitotenv.2020.138226.

[20] J. Shaman and M. Kohn, "Absolute humidity modulates influenza survival, transmission, and seasonality," Proc. Natl. Acad. Sci. U. S. A., vol. 106, no. 9, pp. 3243-3248, 2009, doi: 10.1073/pnas.0806852106.

[21] E. G. Gardner, D. Kelton, Z. Poljak, M. Van Kerkhove, S. Von Dobschuetz, and A. L. Greer, "A case-crossover analysis of the impact of weather on primary cases of Middle East respiratory syndrome," BMC Infect. Dis., vol. 19, no. 1, pp. 1-10, 2019, doi: 10.1186/s12879-019-3729-5.

[22] M. Shoji, K. Katayama, and K. Sano, "Absolute humidity as a deterministic factor affecting seasonal influenza epidemics in Japan," Tohoku J. Exp. Med., vol. 224, no. 4, pp. 251-256, 2011, doi: $10.1620 /$ tjem.224.251.

[23] M. Coccia, "Factors determining the diffusion of COVID-19 and suggested strategy to prevent future accelerated viral infectivity similar to COVID," Sci. Total Environ., vol. 729, p. 138474, 2020, doi: 10.1016/j.scitotenv.2020.138474.

[24] P. Das and R. Horton, "Pollution, health, and the planet: time for decisive action," Lancet, vol. 391, no. 10119, pp. 407-408, 2018, doi: 10.1016/S0140-6736 (17)32588-6.

[25] G. Wang et al., "Persistent sulfate formation from London Fog to Chinese haze," Proc. Natl. Acad. Sci. U. S. A., vol. 113, no. 48, pp. 13630-13635, 2016, doi: 10.1073/pnas.1616540113.

[26] M. Wei et al., "Effects of aerosol pollution on PM2.5-associated bacteria in typical inland and coastal cities of northern China during the winter heating season," Environ. Pollut., vol. 262, p. 114188, 2020, doi: 10.1016/j.envpol.2020.114188.

[27] E. Y. Oh, C. Ansell, H. Nawaz, C. H. Yang, P. A. Wood, and W. J. M. Hrushesky, "Global breast cancer seasonality," Breast Cancer Res. Treat., vol. 123, no. 1, pp. 233-243, 2010, doi: 10.1007/s10549-009-0676-7. 